\title{
The effectiveness of trastuzumab (Herceptin) combined with chemotherapy for gastric carcinoma with overexpression of the c-erbB-2 protein
}

\author{
Christine Rebischung ${ }^{1}$, Raphä̈lle Barnoud ${ }^{2}$, Laetitia Stéfani ${ }^{1}$, Jean-Luc Faucheron ${ }^{3}$, \\ and Mireille Mousseau ${ }^{1}$ \\ ${ }^{1}$ Department of Oncology and Haematology, University Hospital, BP 217-38043 Grenoble Cedex 9, France \\ ${ }^{2}$ Department of Anatomopathology, University Hospital of Grenoble, Grenoble, France \\ ${ }^{3}$ Department of Surgery, University of Grenoble, Grenoble, France
}

\begin{abstract}
The c-erbB-2 protein is overexpressed in $7 \%$ of gastric cancer cases, suggesting that anti-c-erbB-2 antibody therapy (trastuzumab; Herceptin) could be used. We report here a 28year-old woman with metastatic gastric cancer overexpressing c-erbB-2 (3 + strong membrane staining on immunohistochemistry) who was treated with trastuzumab in combination with chemotherapy. A complete response was obtained with a combination of trastuzumab and oxaliplatin and was maintained with trastuzumab alone for 18 months. The patient relapsed and chemotherapy (capecitabine, docetaxel) was combined with the anti-c-erbB-2 antibody. The patient survived for 4 years with metastatic disease controlled for 2 years by immunochemotherapy. We conclude that the combination of trastuzumab and chemotherapy is efficient in the treatment of metastatic gastric carcinoma with overexpression of the c-erbB-2 protein.
\end{abstract}

Key words Gastric cancer - c-erbB-2 protein - Anti-EGF antibody $\cdot$ Trastuzumab

\section{Introduction}

c-erbB-2, located on chromosome 17 , encodes a $185-\mathrm{kDa}$ transmembrane tyrosine kinase receptor, which belongs to the growth factor receptor family. In carcinomas, $c$ $e r b B-2$ acts as an oncogene, mainly because high-level amplification of the gene induces protein overexpression (p185) in the cellular membrane [1]. Identifying these c-erbB-2 abnormalities in breast cancer is of interest, since trastuzumab (Herceptin; Roche Pharma, Neuilly sur Seine, France), a monoclonal antibody against the $c$-erbB-2 gene product, has proved effective in such patients. The c-erbB-2 protein has been found to be overexpressed in gastric carcinoma [2], suggesting that anti-c-erbB-2 antibody therapy could be used.

Offprint requests to: C. Rebischung

Received: February 17, 2005 / Accepted: June 22, 2005
We report the case of a patient presenting with a metastatic gastric cancer which was successfully treated by trastuzumab in combination with chemotherapy.

\section{Case report}

A 28-year-old woman presented with gastric cancer and a tumor of the right ovary. She was referred to the University Hospital of Grenoble for treatment in October 1996.

Subtotal gastrectomy and bilateral adnexectomy were performed. The histopathological conclusion was gastric adenocarcinoma with metastasis in the right ovary. The tumor was staged pT3pN1M1 according to the International Union Against Cancer (UICC) classification. No residual tumor was present after surgery. The serum levels of carcinoembryonic antigen (CEA) were normal. From December 6, 1996, to May 9, 1997, the patient received adjuvant combination chemotherapy with fluorouracil and cisplatin.

Two peritoneal metastatic nodules were removed surgically, one in October 1997 and one in March 1998. No other tumor was found during this surgery.

In March 1999, an abdominal computed tomography (CT) scan was performed because the patient had pain in the right hypochondrium; the CT scan revealed a $3 \times$ $3-\mathrm{cm}$ tumor in the lateral abdominal wall. After complete surgical excision, the patient received 12 cycles of chemotherapy with docetaxel and irinotecan (CPT 11), from May 17, 1999, to January 14, 2000.

In March 2000, a 3-cm peritoneal nodule (responsible for bone lysis on the pelvis) invading the peritoneal incision line and pubic bone was treated with surgery and external irradiation.

In December 2000, she presented again, with multiple abdominal-wall nodules. Immunohistochemistry was performed, and c-erbB-2 overexpression $(3+)$ was detected in the tumoral cells. The third-line regimen 
was a combination of trastuzumab and oxaliplatin: trastuzumab (Herceptin: $4 \mathrm{mg} / \mathrm{kg}$ for the first weekly injection, followed by $2 \mathrm{mg} / \mathrm{kg}$ for the next weekly injections) and oxaliplatin (Eloxatine, Sanofi-Aventis, Paris, France: $100 \mathrm{mg} / \mathrm{m}^{2}$ per injection every other week). Complete remission was observed after 3 months of treatment. Peripheral neuropathy was observed, so oxaliplatin was stopped and trastuzumab was continued alone. The treatment with trastuzumab alone lasted for 18 months.

In July 2002, a CT scan revealed bilateral lung metastases, with three nodules, each less than $1 \mathrm{~cm}$ in diameter, in the upper right and left lobes and in the inferior right lobe. Pelvic examination showed a pelvic mass of 6 $\times 5.5 \times 4 \mathrm{~cm}$ anchored to the sigmoid. A sigmoidectomy was performed and the peritoneal nodules were removed, but the patient refused lung surgery. Six months after the sigmoidectomy, chemotherapy and trastuzumab were resumed, in January 2003, in combination with oxaliplatin because of a $3-\mathrm{cm}$ liver metastasis located in segment V. Oxaliplatin was replaced by capecitabine (Xeloda [Roche] $1000 \mathrm{mg} / \mathrm{m}^{2}$ per day, on days 1 to 14 , every 3 weeks) because of digestive intolerance. The disease was controlled after 12 months of treatment. Stabilization of the lung and liver metastases was confirmed by CT scan, but the patient developed peritoneal carcinomatosis in June 2004. Chemotherapy was switched for docetaxel combined with trastuzumab, but the patient developed a bowel occlusion and died in December 2004.

\section{Histopathology of the primary tumor}

The primary gastric tumor measured $5 \times 3 \mathrm{~cm}$ and was staged pT3 according to the UICC classification. The pathologist diagnosed well-differentiated adenocarcinoma (intestinal type according to Lauren's classification), organized in tubular formations (Fig. 1). Six lymph nodes were metastatic out of the eight resected during the lymph node dissection (stage $\mathrm{pN} 1$ ).

The histological analysis of the right ovary tumor (17 $\times 15 \times 8 \mathrm{~cm}$ ) confirmed that the metastasis came from the primary gastric tumor. No other pelvic metastasis was diagnosed in the left ovary, the uterus, or the omentum.

The pathological analysis of all pelvic and intestinal metastases revealed the presence of adenocarcinomatous cells, originating from the primary gastric tumor.

\section{Immunohistochemistry}

To assess c-erbB-2 expression, the primary gastric tumor was scored according to the criteria recom- mended by Dako for the Herceptest. Dako clone A 485 antibody was used. Interpretation revealed strong $(3+)$ membrane staining (Fig. 2).

\section{Discussion}

In gastric cancer, overexpression of c-erB-2 protein, detected by immunohistochemistry, was first described in 1986 [2]. According to a recent study using fluorescence in situ hybridization (FISH), this overexpression is well correlated with gene amplification and both are detected in approximately $7 \%$ of gastric cancer cases [3], without a clear relationship with histological subtypes. The mechanisms of 185 protein overexpression and of the high-level amplification of $c-e r b B-2$ in gastric cancer are similar to those observed in breast cancer [1].

Patients with a breast tumor showing Her-2 gene amplification and protein overexpression have an inferior prognosis, with shorter disease-free and overall survivals $[1,4]$. The prognostic significance of Her-2/ neu overexpression was studied in gastric carcinoma. An especially low survival probability was reported for tumor-resected patients with an immunohistochemical c-erbB-2 score of 2 or 3 [5]; their overall survival was 29 months vs 42 months for those with a tumor negative for c-erbB-2. In our study, the expression of the $c$-erb $B-2$ gene appeared as an independent biological risk factor, and a significant association between the expression of Her-2/neu and proteases involved in invasion and metastasis was observed. In gastric cancer, c-erbB-2 overexpression seems to be the second poorest prognostic variable after nodal status [6], but its prognostic role is significant only in early-stage tumors [7].

Little is known about the prognostic value of c-erbB2 overexpression in metastatic gastric carcinoma. In metastatic breast cancer, trastuzumab significantly enhances the activity of first-line chemotherapy and enhances survival in women with Her-2-overexpressing breast cancer [8]. In the same way, gastric adenocarcinomas with strong membrane staining $(3+)$ are potential targets for this new treatment. The effectiveness of this c-erbB-2 targeting antibody in the inhibition of the growth of gastric cancer cell lines was recently reported [9], but, to our knowledge, trastuzumab has not yet been studied systematically as an indication for c-erbB-2 protein overexpressing gastric cancer.

In the present patient, the tumor was a metastatic well-differentiated gastric adenocarcinoma, and showed strong $(3+)$ membrane staining on immunohistochemistry. The patient was efficiently treated with the combination of trastuzumab and oxaliplatin, which are effective drugs in the treatment of gastric cancer, and a 

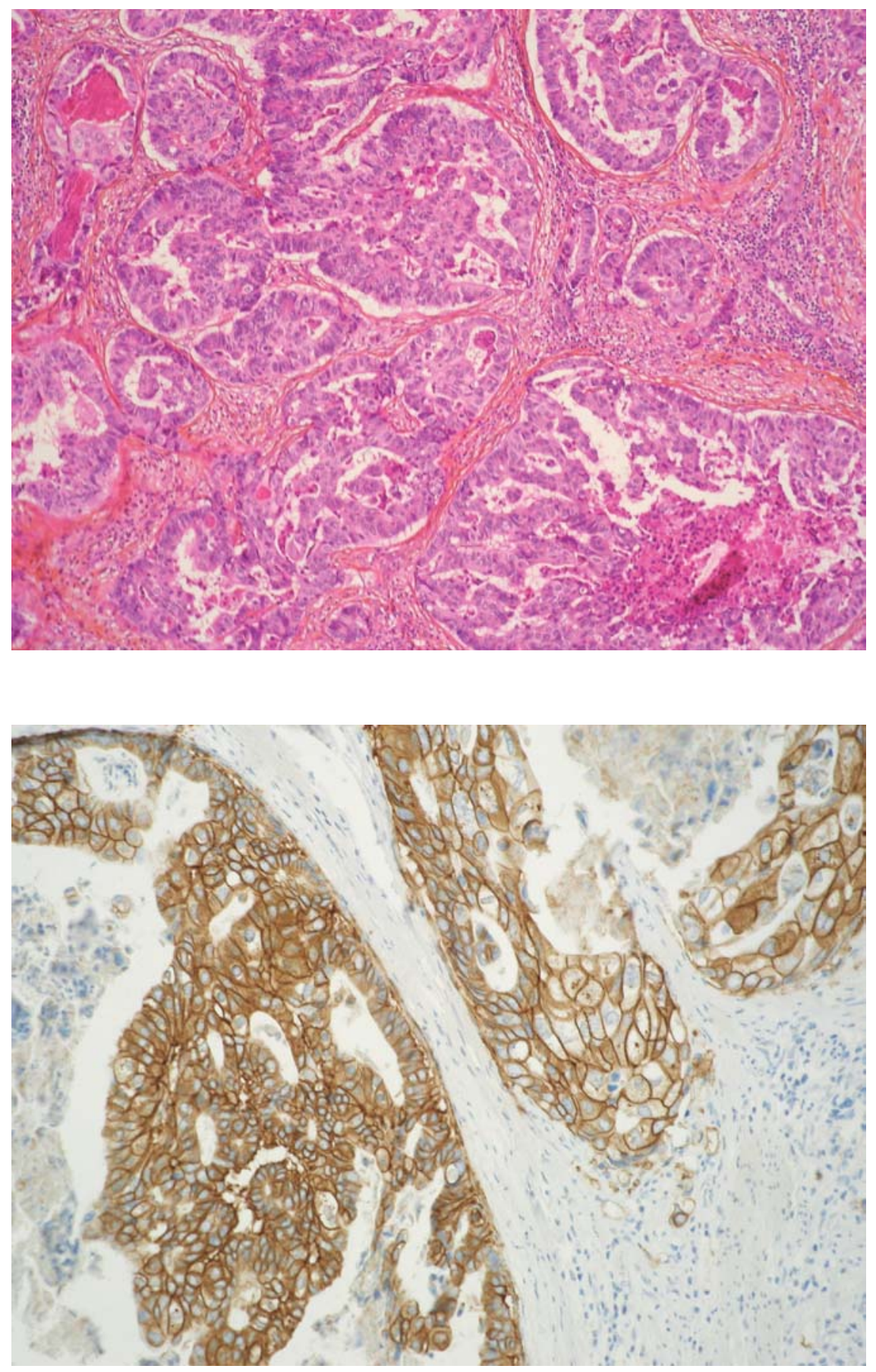

Fig. 1. Histological analysis of the gastric tumor revealed a well-differentiated adenocarcinoma, organized in tubular formations. HES staining, $12.5 \times 4$
Fig. 2. Immunohistochemistry of the gastric tumor revealed strong $(3+)$ membrane c-erbB-2 staining. $12.5 \times 10$ complete response to peritoneal carcinomatosis was obtained. In a recent study, oxaliplatin in combination with 5-fluorouracil (5FU) and folinic acid led to a response rate of $45 \%$ for this tumor type [10]. Our patient developed grade 3 neurotoxicity, due to oxaliplatin, and trastuzumab was then given alone. Lung and peritoneal metastases developed despite the therapy. The disease was controlled again after reintroducing chemotherapy which had proved effective in the treatment of gastric cancer (oxaliplatin, then capecitabine) [10,11]. The combination of trastuzumab and chemotherapy allowed for better disease control than immunotherapy alone, and an additive effect was suspected for the gastric tumor. In breast cancer, the combination of trastuzumab and chemotherapy is synergistic. Treatment with anthracycline, cyclophosphamide, paclitaxel, or vinorelbine, and trastuzumab is more efficient than monotherapy, with the objective response rate increasing from $26 \%$ to $50 \%$, and an improved overall survival (25.1 vs 20.3 months; $P=0.046)[12,13]$. 
The median survival of patients with metastatic gastric cancer treated by chemotherapy alone is 9 months [14]. In contrast, our patient survived for 4 years with metastatic disease controlled for 2 years by immunochemotherapy. This extended survival, perhaps favored by unexpectedly weak activity of the tumor, suggests that it will be of high interest to further evaluate the combination of trastuzumab and chemotherapy in the treatment of gastric carcinoma with overexpression of the c-erbB-2 protein.

\section{References}

1. Slamon DJ, Godolphin W, Jones LA, Holt JA, Wong SG, Keith DE, et al. Studies of the HER-2/neu proto-oncogene in human breast and ovarian cancer. Science 1989;244:707-12.

2. Sakai K, Mori S, Kawamoto T, Taniguchi S, Kobori O, Morioka $\mathrm{Y}$, et al. Expression of epidermal growth factor receptors on normal human gastric epithelia and gastric carcinomas. J Natl Cancer Inst 1986;77:1047-52.

3. Takehana T, Kunitomo K, Kono K, Kitahara F, Iizuka H, Matsumoto Y, et al. Status of c-erbB-2 in gastric adenocarcinoma: a comparative study of immunohistochemistry, fluorescence in situ hybridization and enzyme-linked immuno-sorbent assay. Int $\mathbf{J}$ Cancer 2002;98:833-7.

4. Ravdin PM, Chamness GC. The c-erbB-2 proto-oncogene as a prognostic and predictive marker in breast cancer: a paradigm for the development of other macromolecular markers - a review. Gene 1995;159:19-27.

5. Allgayer H, Babic R, Gruetzner KU, Tarabichi A, Schildberg FW, Heiss MM. C-erbB-2 is of independant prognostic relevance in gastric cancer and is associated with the expression of tumorassociated protease systems. J Clin Oncol 2000;18:2201-9.
6. Nakajima M, Sawada H, Yamada Y, Watanabe A, Tatsumi M, Yamashita $\mathrm{J}$, et al. The prognostic significance of amplification and overexpression of c-met and c-erbB-2 in human gastric carcinomas. Cancer 1999;85:1894-902.

7. Mizutani T, Onda M, Tokunaga A, Yamanaka N, Sugisaki Y. Relationship of c-erbB-2 protein expression and gene amplification to invasion and metastasis in human gastric cancer. Cancer 1993;72:2083-8.

8. Vogel CL, Cobleigh MA, Tripathy D, Gutheil JC, Harris LN, Fehrenbacher L, et al. Efficacy and safety of trastuzumab as a single agent in first-line treatment of HER2-overexpressing metastatic breast cancer. J Clin Oncol 2002;20:719-26.

9. Tanner M, Hollmen M, Juntilla TT, Kapanen AI, Tommola S, Soini Y, et al. Amplification of Her-2 in gastric carcinoma: association with topoisomerase II $\alpha$ gene amplification, intestinal type, poor prognosis and sensitivity to trastuzumab. Ann Oncol 2005; 16:273-8.

10. Louvet C, Andre T, Tigaud JM, Gamelin E, Douillard JY, Brunet $\mathrm{R}$, et al. Phase II study of oxaliplatin, fluorouracil, and folinic acid in locally advanced or metastatic gastric cancer patients. J Clin Oncol 2002;20:4543-8.

11. Evans TR, Pentheroudakis G, Paul J, McInnes A, Blackie R, Raby N, et al. A phase I and pharmacokinetic study of capecitabine in combination with epirubicin and cisplatin in patients with inoperable oesophago-gastric adenocarcinoma. Ann Oncol 2002;13:1469-78.

12. Slamon DJ, Leyland-Jones B, Shak S, Fuchs H, Paton V, Bajamonde A, et al. Use of chemotherapy plus a monoclonal antibody against HER2 for metastatic breast cancer that overexpresses HER2. N Engl J Med 2001;344:783-92.

13. Burstein HJ, Kuter I, Campos SM, Gelman RS, Tribou L, Parker LM, et al. Clinical activity of trastuzumab and vinorelbine in women with Her2-overexpressing metastatic breast cancer. J Clin Oncol 2001;19:2722-30.

14. Webb A, Cunningham D, Scarffe JH, Harper P, Norman A, Joffe $\mathrm{JK}$, et al. Randomized trial comparing epirubicin, cisplatin, and fluorouracil versus fluorouracil, doxorubicin, and methotrexate in advanced esophagogastric cancer. J Clin Oncol 1997;15:1-7. 